\title{
Asian forum on environmental health policy: challenges and perspectives of environmental health problems in the region in the next 30 years
}

\author{
Kyungho Choi - Domyung Paek - Tangchun Wu \\ Chang-Chuan Chan $\cdot$ Rattapon Onchang • \\ Chantana Padungtod · Akio Koizumi
}

Published online: 16 February 2012

(C) The Japanese Society for Hygiene 2012

\section{Introduction}

Asia is the world's most populous continent, accomodating circa 3.9 billion people in 48 countries. It is an important player in terms of the environment and world economy, with the second largest gross domestic product of all continents, after Europe. This geographical entity includes many developed countries, such as Japan, South Korea, and Singapore, and a number of very high growth nations, such as China and India. Its climate is very diverse, ranging from the very moist (southeastern parts) to dry (interior), and very cold (e.g., Siberia) to tropical (southern regions). The countries of this continent not only share-to varying degrees-historical experiences but also the effects of

\section{K. Choi $(\square) \cdot$ D. Paek}

School of Public Health, Seoul National University,

Seoul, Korea

e-mail: kyungho@snu.ac.kr

T. Wu

School of Public Health, Tongji Medical College,

Huazhong University of Science and Technology, Hubei, China

C.-C. Chan

College of Public Health, National Taiwan University,

Taipei, Taiwan

R. Onchang

Faculty of Science, Silpakorn University,

Nakhon Pathom, Thailand

C. Padungtod

Department of Disease Control, Ministry of Public Health,

Nonthaburi, Thailand

A. Koizumi

School of Public Health, Graduate School of Medicine,

Kyoto University, Kyoto, Japan environmental pollution, such as Asian dust storms (ADS). The recent Fukushima Daiichi nuclear disaster in Japan has delivered the clear message that although an environmental challenge may be initiated in one specific location, many countries in the region may suffer from the consequences. Global climate change also underlines the fact that environmental issues are often global and, consequently, multinational collaboration is not an option but a necessity. Environmental health problems cannot be properly resolved if the counter-measures are restricted only to within the political borders. Accurate assessment of the problems and the development of appropriate solutions can best be achieved on a broader, regional scale, by communication and collaboration among those scientists and policy-makers facing the same issues.

The past of one country can mirror the present or future situation of another country. Japan, one of the most developed countries in the world, and South Korea, now considered to be a developed country, have experienced very fast economic growth, which has been accompanied by worsening health of its population due to environmental pollution. Ageing has for some time been an important issue in Japanese society and is now also a challenging problem in South Korea. China is the largest economy in Asia and the second largest economy in the world. However, it is now recognizing the adverse consequences of rapid industrialization and development in terms of environmental health. The experiences of South Korea or Japan could help China better prepare for the potential challenges to be faced in terms of environmental health.

Such recognition has led to the organization of a longterm platform for communication and collaboration among environmental health experts representing the diverse geographical areas of Asia. The first International Forum for Environmental Health Policy and Science was held in 
Seoul in November 25, 2011, and delegates from leading academic institutes in Bangkok, Kyoto, Seoul, Taipei, and Wuhan participated. The editorial presents a summary of the motivation for holding this forum, the perspectives of each participant, and future directions that were agreed on, with the intention to encourage other scientists around the region to join in and contribute.

\section{Issues and agendas by delegation}

Japan

First of all, we deeply express our sincere thanks for the warm support we have received from Asian countries that may have been directly or indirectly affected by the consequences of the East Japan Earthquake. We also have to admit that serious environmental contamination did take place due to the escape of radionuclides from the crippled Fukushima Daiichi nuclear power plant. International environmental monitoring for radionuclides will be needed for the next 30 years. In addition, we have urged the Japanese government to make information on the crippled plant accessible to the international community.

In the current, rather complex global circumstances, policy-making by any one nation on trans-boundary environmental problems cannot be independent of national interests in making profits because the latter are closely linked to the national economy. However, it is obvious that too much emphasis on the national economy when challenged with trans-boundary environmental problems will only increase conflicts among neighboring countries. Thus, we need to find a way to harmonize policy-making among countries and should establish clear and simple mechanisms to delineate the responsibility of stakeholder countries. One such a way is to visualize the trans-boundary flows of environmental contaminants among Asian countries. Such visualization will increase the transparency to the general populations of Asian countries, facilitating decision-making on the basis of national consensus. The Asian platform is expected to function as a task force for the visualization.

\section{Korea}

Demands for a cleaner and healthier environment have become very high in South Korea after several decades of rapid industrialization and development. These demands reflect the increased awareness among the general public of the potential deleterious health effects from exposure to environmental contamination. While serious environmental pollution events due to industrial incidents or by accidental release have since the mid-1990s no longer been frequently occurred, recent experiences, such as the Hebei-Spirit oil spill of 2007, still demonstrate the potential for such events to occur at any time. Therefore, the importance of emergency preparedness cannot be emphasized enough within the framework of environmental health management. In addition to such accidental episodes, the most important health issues related to environmental problems include the consequences of long-term low-dose exposures to multiple environmental contaminants, and environmental health inequity among susceptible populations, such as children and the elderly. Emerging environmental issues associated with global climate change and new technologies (e.g., nanomaterials) also deserve special attention. The Center for Disease Control of South Korea recently reported that the epidemic of acute interstitial pneumonia and the several resulting casualties were likely the result of exposure to a number of disinfectants used in humidifiers. This incidence increased public skepticism on the safety of chemicals that are used in normal daily activities. However, it also provides a chance to critically review the systems for ensuring chemical safety, not only before the release of such chemicals onto the market, but also during the marketing period.

\section{China}

China is the world's fastest growing major economy, with annual growth rates of approximately $10 \%$ for almost three decades. Since the 1980 s China has witnessed increased pollution and degradation of natural resources and now recognizes increasing public health problems due to such pollution. Chronic, non-communicable diseases account for about $80 \%$ of deaths and $70 \%$ of disability-adjusted lifeyears lost in China, which is in part related to changes in the environment, lifestyle and diet, as well as an increased life expectancy. Air pollution has emerged as the most important environmental cause of cardiopulmonary diseases and adverse health risks. Lifestyle and diet changes, environmental pollution, and their interaction with genetics or epigenetic factors are involved in endemic, chronic noncommunicable diseases. One of the major scientific challenges for the next few decades will be to gain an understanding of the interaction between genetic susceptibility and environmental factors on the etiology of not only cardiopulmonary diseases, such as heart disease, asthma, lung cancer, and chronic obstructive pulmonary diseases, but also on early damage, such as genetic damage, decreased lung function, and heart rate variability. Environment-wide and genome-wide association studies on chronic non-communicable diseases can best be carried out by collaborations and through the exchange of ideas within the region that shares common environmental problems. 
Therefore, we are looking forward to future cooperation in Asia.

Taiwan

Asian dust storms occur in the winter and spring, especially from March to May, and mainly originate in the Gobi and Takla Makan deserts of Mongolia and western China. They can move eastward to China, Japan, South Korea, Taiwan, and sometimes to northern Pacific Ocean areas. Particulate matter (PM), especially PM with aerodynamic diameters of $<10 \mu \mathrm{m}\left(\mathrm{PM}_{10}\right)$, have been recorded at concentrations of $>500 \mu \mathrm{g} / \mathrm{m}^{3}$, occasionally even exceeding $1,000 \mu \mathrm{g} / \mathrm{m}^{3}$, during several ADS in many cities located downwind of these deserts, including Shanghai, Hong Kong, and Taipei. Overall findings of epidemiological studies show that longrange transported Asian dust can increase mortality among residents in downwind areas. Dust derived from mineral soil in deserts and air pollutants from biomass burning are major contributors of long-range transported air pollution across many countries around the world. The negative effects of trans-boundary air pollution on public health are emerging as an important global health issue which needs to be further researched by academics and governments. Global efforts, including alleviating desertification speed in dust-originating areas and reducing industrial emissions along the dust-transporting paths, must be made to tackle the root-causes of trans-boundary air pollution in order to protect the global environment and public health. Collaboration among Asian public health researchers to tackle this trans-boundary pollution problem is recommended as a major step towards protecting public health and environmental quality in this fast growing continent.

\section{Thailand}

Thailand is a newly industrialized country and one of the fastest growing economies, ranked 24th on the global market. Thailand is now experiencing environmental issues as a downside of economic growth, which include deforestation and air pollution, among many others. Assessment of health damage due to industrialization and associated remediation approaches are important in Thailand. Climate change, which may affect human health through a range of mechanisms, including the relatively direct risks of floods and storms and the more complex pathways of altered patterns of infectious disease outbreak, is a growing concern of Thailand, and one that might be appropriately addressed by collaborative efforts within Asian countries. Scientific research on common environmental issues, such as trans-boundary air pollution and climate change, is essential given the nature of the problems. It is also important to encourage community empowerment by, for example, organizing practical workshops aimed at distributing research findings to the community or by initiating local-based research activities in accordance with their most critical environmental problems.

\section{Consensus and future directions}

We have reached a consensus on the long- and short-term objectives of the forum. As the long-term objective, we should collaborate more closely on Asian environmental health problems at several levels-individual scientist level, academic society level, and governmental level. We believe that collaborations among academic societies can provide the greatest impulse. In pursuing the short-term goals, we agreed it would be important to develop or foster a high-quality scientific journal in environmental health. Environmental Health and Preventive Medicine (EHPM) is obviously one of the most potential candidate journals for this role.

To identify priority environmental health issues in the region and to develop a collaborative research network to resolve the challenges, we agreed to organize a steering committee for the Asian Environmental Health Forum, the members of which will be chosen from among the delegates from Asian countries. The committee will support policy-makers in each country by providing knowledge and sharing experiences from other countries. It will also play a pivotal role in identifying those areas warranting the attention of policy-makers and provide channels of communication among policy-makers of countries that share or have shared similar challenges.

To form a steering committee for the forum and to identify environmental health issues in the region that should be given priority in terms of policy, the Korean Society of Environmental Health will take the initiative to organize a series of annual workshops in collaboration with Ministry of Environment of Korea. We hope that the initiative will promote academic communication and identify ideas for synergistic collaborations on environmental health issues during the next 30 years. 